Original article

\title{
Pathogenic potential of a Costa Rican strain of 'Candidatus Rickettsia amblyommii' in guinea pigs (Cavia porcellus) and protective immunity against Rickettsia rickettsii
}

\author{
Juan J. Rivas ${ }^{\mathrm{a}}$, Andrés Moreira-Soto ${ }^{\mathrm{a}, \mathrm{b}}$, Gilberth Alvarado ${ }^{\mathrm{c}, \mathrm{d}}$, Lizeth Taylor ${ }^{\mathrm{a}, \mathrm{b}, 1}{ }^{\text {, }}$ \\ Olger Calderón-Arguedas ${ }^{\mathrm{a}, \mathrm{e}}$, Laya Hun ${ }^{\mathrm{a}, \mathrm{b}}$, Eugenia Corrales-Aguilar ${ }^{\mathrm{a}, \mathrm{b}}$, \\ Juan Alberto Morales ${ }^{\mathrm{f}}$, Adriana Troyo ${ }^{\mathrm{a}, \mathrm{e}, *}$ \\ a Centro de Investigación en Enfermedades Tropicales, Universidad de Costa Rica, San José, Costa Rica \\ b Sección de Virología, Facultad de Microbiología, Universidad de Costa Rica, San José, Costa Rica \\ c Centro de Investigación en Estructuras Microscópicas, Universidad de Costa Rica, San José, Costa Rica \\ d Escuela de Biología, Universidad de Costa Rica, San José, Costa Rica \\ e Sección de Entomología Médica, Facultad de Microbiología, Universidad de Costa Rica, San José, Costa Rica \\ ${ }^{\mathrm{f}}$ Servicio de Patología, Escuela de Medicina Veterinaria, Universidad Nacional, Heredia, Costa Rica
}

\section{A R T I C L E I N F O}

\section{Article history:}

Received 2 March 2015

Received in revised form 3 July 2015

Accepted 13 July 2015

Available online $\mathrm{xxx}$

\section{Keywords:}

Rickettsia amblyommii

Experimental infection

Immunity

Guinea pig

Costa Rica

\begin{abstract}
A B S T R A C T
'Candidatus Rickettsia amblyommii' is a spotted fever group rickettsia that is not considered pathogenic, although there is serologic evidence of possible infection in animals and humans. The aim of this study was to evaluate the pathogenic potential of a Costa Rican strain of 'Candidatus R. amblyommii' in guinea pigs and determine its capacity to generate protective immunity against a subsequent infection with a local strain of Rickettsia rickettsii isolated from a human case. Six guinea pigs were inoculated with 'Candidatus R. amblyommii' strain 9-CC-3-1 and two controls with cell culture medium. Health status was evaluated, and necropsies were executed at days 2, 4, and 13. Blood and tissues were processed by PCR to detect the gltA gene, and end titers of anti-'Candidatus R. amblyommii' IgG were determined by indirect immunofluorescence. To evaluate protective immunity, another 5 guinea pigs were infected with 'Candidatus R. amblyommii' (IGPs). After 4 weeks, these 5 IGPs and 3 controls (CGPs) were inoculated with pathogenic $R$. rickettsii. Clinical signs and titers of anti-Rickettsia IgG were determined. IgG titers reached 1:512 at day 13 post-infection with 'Candidatus R. amblyommii'. On day 2 after inoculation, two guinea pigs had enlarged testicles and 'Candidatus R. amblyommii' DNA was detected in testicles. Histopathology confirmed piogranulomatous orchitis with perivascular inflammatory infiltrate in the epididymis. In the protective immunity assay, anti-Rickettsia IgG end titers after $R$. rickettsii infection were lower in IGPs than in CGPs. IGPs exhibited only transient fever, while CGP showed signs of severe disease and mortality. $R$. rickettsii was detected in testicles and blood of CGPs. Results show that the strain 9-CC-3-1 of 'Candidatus R. amblyommii' was able to generate pathology and an antibody response in guinea pigs. Moreover, its capacity to generate protective immunity against $R$. rickettsii may modulate the epidemiology and severity of Rocky Mountain spotted fever in areas where both species circulate.
\end{abstract}

(c) 2015 Elsevier GmbH. All rights reserved.

\section{Introduction}

'Candidatus R. amblyommii' was first detected in Amblyomma americanum and referred to as the WB-8-2 Rickettsia (Burgdorfer et al., 1981). In these studies, Burgdorfer

\footnotetext{
* Corresponding author at: Departamento de Parasitología, Facultad de Microbiología, Universidad de Costa Rica, San José 11501, Costa Rica.

E-mail address: adriana.troyo@ucr.ac.cr (A. Troyo).

1 Deceased (December, 2013).
}

http://dx.doi.org/10.1016/j.ttbdis.2015.07.008

1877-959X/@ 2015 Elsevier GmbH. All rights reserved. et al. concluded that WB-8-2 Rickettsia was probably not pathogenic for humans because it did not generate an immune response or disease in guinea pigs, although an antibody response was evidenced in field mice (Microtus pennsylvanicus) (Burgdorfer et al., 1981). 'Candidatus R. amblyommii' has since been identified as a common Rickettsia in ticks of North and South America, especially those of the genus Amblyomma (Mixson et al., 2006; Labruna et al., 2011). In some areas, its prevalence in ticks can be higher than 50\% (Bermúdez et al., 2009; Jiang et al., 2010; Zhang et al., 2012; Blanton et al., 2014; Nadolny et al., 2014). 
The pathogenic potential of 'Candidatus R. amblyommii' has been debated. For example, a localized rash was attributed to 'Candidatus R. amblyommii' after a tick bite (Billeter et al., 2007), and seroconversion with a fourfold or greater rise in IgG titers to 'Candidatus R. amblyommii', but not to Rickettsia rickettsii, was demonstrated in patients with a presumptive clinical diagnosis of Rocky Mountain spotted fever (RMSF) in North Carolina, USA (Apperson et al., 2008). In Tennessee, where cases of RMSF are frequently reported, a study failed to find $R$. rickettsii in ticks, but found a high prevalence of 'Candidatus R. amblyommii' in $A$. americanum (Moncayo et al., 2010). There is also molecular and serological evidence of 'Candidatus R. amblyommii' infection in dogs following exposure to tick bites (Barrett et al., 2014), while other seroprevalence studies also suggest that there may be infection in dogs and horses (Labruna et al., 2007; Bermúdez et al., 2011). However, a recent study failed to detect signs of disease in guinea pigs infected with a North American strain of 'Candidatus R. amblyommii', confirming Burgdorfer's initial finding (Blanton et al., 2014).

Several rickettsiae are known to elicit an immune response that may later protect its host from a more pathogenic species. This has been demonstrated for species such as Rickettsia montanensis, Rickettsia australis, Rickettsia conorii, Rickettsia typhi, and more recently for 'Candidatus R. amblyommii' (Feng and Waner, 1980; Walker et al., 1984; Feng and Walker, 2003; Blanton et al., 2014). Considering that strains of rickettsiae may show differences in virulence and that 'Candidatus R. amblyommii' and $R$. rickettsii are present in many areas of Central and South America (Ellison et al., 2008; Parola et al., 2013), the purpose of this study was to evaluate the pathogenic potential of a Costa Rican strain of 'Candidatus R. amblyommii' in guinea pigs and confirm its capacity to generate cross-protective immunity against a local virulent strain of $R$. rickettsii.

\section{Materials and methods}

\subsection{Animals}

Male guinea pigs, Cavia porcellus, 200-280 g body weight, were used at the beginning of all experiments. They were maintained in separate cages at the Animal Research Laboratory of Universidad de Costa Rica with vitamin C supplement, and food and water ad libitum. When indicated for each experiment, animals were anesthetized with an intramuscular injection (dosage $25 \mathrm{mg} / \mathrm{kg}$ ) of Zoletil ${ }^{\circledR} 50$ (Virbac), which is a mixture of tiletamine and zolazepam ( $25 \mathrm{mg} / \mathrm{mL}$ of each). To euthanize animals, an overdose of these anesthetics was applied, followed by an intracardiac injection of magnesium sulfate. All experiments and procedures were performed or supervised by a veterinarian, were approved by Universidad de Costa Rica's Institutional Committee for the Use and Care of Laboratory Animals (number CICUA-35-10), and follow the International Guiding Principles for Biomedical Research Involving Animals (CIOMS and ICLAS, 2012).

\subsection{Rickettsia isolates}

'Candidatus R. amblyommii' strain 9-CC-3-1 was isolated in Costa Rica from Amblyomma cajennense sensu lato (Hun et al., 2011). The pathogenic isolate of $R$. rickettsii employed was obtained from a human case (Arguello et al., 2012). Both rickettsiae were cultured separately in confluent monolayers of Vero E6 cells in Minimal Essential Medium (MEM) supplemented with 5\% fetal bovine serum and maintained at $28{ }^{\circ} \mathrm{C}$ and $5 \% \mathrm{CO}_{2}$. The second passage of 'Candidatus R. amblyommii' strain 9-CC-3-1 and the third passage of $R$. rickettsii were used. Unless otherwise stated, the concentration of bacteria was determined by flow cytometry using an acridine orange staining $(0.01 \mu \mathrm{g} / \mathrm{mL})$ with a Guava EasyCyte ${ }^{\mathrm{TM}}$ cytometer (Silverman et al., 1979; Luce-Fedrow et al., 2012).

\subsection{Pathogenic potential of 'Candidatus R. amblyommii'}

A total of eight guinea pigs were used to assess pathogenic potential. The method for infection and evaluation of guinea pigs was adapted from those described previously with other rickettsiae (Feng and Waner, 1980). On day 0, all animals were anesthetized, and 6 guinea pigs were inoculated intraperitoneally with $1 \mathrm{~mL}$ of $4 \times 10^{6}$ 'Candidatus $\mathrm{R}$. amblyommii' bacteria suspended in MEM. The approximate concentration of bacteria was determined with Breed's method by performing dilutions and counting bacteria stained with Giménez stain under a light microscope (1000× magnification) (Giménez, 1964) and by acridine orange staining with a flow cytometer (see above). Two other guinea pigs served as controls and were inoculated in the same manner with only MEM.

Weight, temperature, and clinical signs of disease were evaluated for all guinea pigs at days $0,1,2,3,4,7,9$, and 11 . Animals were anesthetized and a $0.1 \mathrm{~mL}$ blood sample was drawn by cardiac puncture on days $0,1,2,3,4,7,9,11$, and 13 . Serum and blood clot were separated and stored at $-20^{\circ} \mathrm{C}$ for immunofluorescence and PCR analyses, respectively. Necropsies of infected guinea pigs were performed in duplicate on days 2,4 , and 13 . Necropsies of both controls were done on day 13 . The last day of the experiment was determined based on Feng and Waner (1980) and when no increase was detected in IgG end point titers by immunofluorescence, as well as by continuous negative PCR results on previous days. Tissue samples from brain, heart, lungs, spleen, liver, intestines, kidneys, and testicles of each guinea pig were stored frozen at $-20^{\circ} \mathrm{C}$ for PCR analyses or preserved in $10 \%$ buffered formalin for histopathological studies. Organs were processed by standard histopathological protocols, and organ sections were stained with hematoxylin-eosin (H\&E).

\subsection{Cross-protective immunity}

A total of eight guinea pigs were used to assess cross-protective immunity. The method for evaluating cross-protective immunity in guinea pigs was adapted from the one described by Feng and Waner (1980). Five guinea pigs (IGPs) were inoculated intraperitoneally on day 0 with $6 \times 10^{6}$ 'Candidatus R. amblyommii' bacteria suspended in $1 \mathrm{~mL}$ of MEM. Another 3 guinea pigs were inoculated with MEM and used as non-immune controls (CGPs). At day 0 and for the following three weeks, temperature, weight, and signs of disease were evaluated twice weekly. A $0.1 \mathrm{~mL}$ blood sample was drawn once a week by cardiac puncture to detect anti-Rickettsia IgG by immunofluorescence.

One month later (day 32 after initial infection), IGPs and CGPs were infected intraperitoneally with $1 \mathrm{~mL}$ of $1 \times 10^{6} \mathrm{R}$. rickettsii, which is equal to the $50 \%$ tissue culture infectious dose (TCID 50 ). The $\mathrm{TCID}_{50}$ for the pathogenic strain of $R$. rickettsii was determined in Vero E6 cells with the Dulbecco plaque assay, according to methods described previously (Wike et al., 1972). Temperature, weight, and signs of disease were evaluated daily until the end of the experiment on day 11 after $R$. rickettsii infection, when all animals were euthanized simultaneously. The end of the experiment was determined by the need to euthanize 2 CGPs following the recommendation of the veterinarian in charge, who established that the animals were moribund.

Two sample $t$-tests were used to determine the statistical significance of differences in mean temperature and weight changes between IGPs and CGPs on the last day of the experiment $(0.05$ level). The variation introduced due to differences in the initial weight of guinea pigs was corrected by subtracting the weight of 
each guinea pig on day 0 from the values obtained in the following evaluations.

Blood samples $(0.1 \mathrm{~mL})$ were drawn from all guinea pigs by cardiac puncture on the day of $R$. rickettsii inoculation and during necropsies. Sera and blood clots were separated and stored at $-20^{\circ} \mathrm{C}$ for immunofluorescence and PCR analyses, respectively. Necropsies were performed, and tissue samples from brain, heart, lungs, spleen, liver, intestines, kidneys and testicles of each guinea pig were frozen at $-20^{\circ} \mathrm{C}$ until analyzed by PCR.

\subsection{PCR analyses and indirect immunofluorescence}

For PCR analyses, genomic DNA was extracted from blood clots and tissue samples using the NucleoSpin ${ }^{\circledR}$ Tissue kit (Macherey-Nagel), following the manufacturer's instructions. Rickettsia-specific DNA was detected by single-step PCR using primers CS-78 and CS-323 of the gltA gene ( $401 \mathrm{bp}$ product) (Labruna et al., 2004). Presence of the ompA gene was determined with primers $\operatorname{Rr} 190-70$ and $\operatorname{Rr} 190-701$ and a subsequent seminested step with primers $\operatorname{Rr} 190-70$ and $\operatorname{Rr} 190-602$ that yield a 532 bp product (Regnery et al., 1991; Roux et al., 1996). The $R$. rickettsii isolate was used as a positive control and C6/36 or Vero E6 cells as a negative control. Products were visualized under ultraviolet light after being run in a $2 \%$ agarose gel.

The presence of anti-Rickettsia sp. IgG antibodies in guinea pig sera was determined by indirect immunofluorescence using 'Candidatus R. amblyommii' and $R$. rickettsii antigen, according to methods described previously (Walker et al., 1977). Anti-guinea pig IgG produced in goat and conjugated to fluorescein isothiocyanate (FITC) (Sigma-Aldrich) was used as secondary antibody at a dilution of $1: 128$. Each sample was first evaluated at dilution of $1: 32$, and an end point titer was determined in two-fold serial dilutions of positive samples. Controls included were anti-Rickettsia sp. IgG positive and negative guinea pig sera.

\section{Results}

\subsection{Pathogenic potential of 'Candidatus R. amblyommii'}

Two days after inoculation with 'Candidatus R. amblyommii' strain 9-CC-3-1, two of six guinea pigs showed bilateral enlargement of the testicles upon clinical examination. These were the two guinea pigs euthanized on day 2 . None of the other infected animals presented clinical signs of disease when compared to control guinea pigs. Infected and uninfected animals gained weight in a similar manner throughout the experiment, and temperature stayed within the normal range $\left(37.5-39.5^{\circ} \mathrm{C}\right)$ (data not shown).

During the necropsies, both of the guinea pigs euthanized on day 2 had enlarged testicles, as did one of the guinea pigs euthanized on day 4. Histologically, the testicles of one guinea pig sacrificed on day 2 showed an inflammatory process around testis with a perivascular infiltrate of polymorphonuclear cells (predominant) and some mononuclear cells (Fig. 1A). In addition, the epididymis showed a perivascular inflammatory infiltrate with mainly mononuclear cells (Fig. 1B). The testicles of the second guinea pig euthanized on day 2 presented a severe inflammatory process into testis with an infiltration consisting mainly of polymorphonuclear cells and some mononuclear cells (Fig. 1C); there was also vascular congestion and extravasation of erythrocytes. The epididymis showed a perivascular inflammatory infiltrate mainly with mononuclear cells (Fig. 1D). The guinea pig with enlarged testicles euthanized on day 4 had a light infiltration of polymorphonuclear cells and some mononuclear cells in the tunica albuginea without testicular lesions (Fig. 1E, $1 \mathrm{~F})$. No other significant gross or microscopic alterations that differed between control and infected animals were observed after necropsies and pathologic evaluations of all guinea pigs analyzed.

Of all the organs analyzed by PCR, the presence of 'Candidatus R. amblyommii' was evidenced by detection of the gltA and ompA fragments only in the testicles of the two guinea pigs euthanized on day 2.

Anti-'Candidatus R. amblyommii' IgG was detected in all infected guinea pigs on day 7 with an end point titer of 1:32. Antibody titer reached a maximum of 1:512 on day 11 in all guinea pigs evaluated, and remained constant until the end of the experiment on day 13 (Fig. 2). Cross-reactivity with $R$. rickettsii antigen was detected on day 9 ( $1: 32$ titer), and the end point titer increased to a maximum of 1:128 (Fig. 2). All guinea pigs showed the same end point titers on each day evaluated.

\subsection{Cross-protective immunity}

All guinea pigs infected with 'Candidatus R. amblyommii' strain 9-CC-3-1 (IGPs) prior to their inoculation with $R$. rickettsii developed IgG antibodies to antigen of 'Candidatus R. amblyommii' with a maximum end point titer of 1:512 (Fig. 3). IGPs also had crossreacting $\operatorname{IgG}$ end point titers of 1:128 to antigen of $R$. rickettsii. No weight loss, fever, or apparent disease was evident in IGPs after inoculation with 'Candidatus R. amblyommii' or in any of the guinea pigs before inoculation with $R$. rickettsii.

Three days after $R$. rickettsii inoculation, one IGP showed moderately enlarged testicles, while all control guinea pigs (CGPs) presented severe bilateral orchitis. Guinea pigs from both groups decreased or stopped weight gain after $R$. rickettsii inoculation; IGPs continued weight gain after 4 to 5 days, but CGPs began losing weight and continued weight loss until the end of the experiment on day 11 after $R$. rickettsii infection (Fig. 4A). On the last day of evaluation, the mean weight change in IGPs and CGPs was $272 \mathrm{~g}$ (95\% CI: 227-317 g) and $148 \mathrm{~g}$ (95\% CI: 49-247 g), respectively, and this difference was statistically significant $(T=4.53 ; p=0.004)$. With only one exception, IGPs showed fever $\left(>39.5^{\circ} \mathrm{C}\right)$ that began $3-5$ days after $R$. rickettsii infection (Fig. 4B) and reached maximum temperatures that ranged from $39.8^{\circ} \mathrm{C}$ to $40.5^{\circ} \mathrm{C}$. Fever lasted 1 day in two IGPs and 5 days in the other two IGPs. All CGPs presented high fever that began on day 4 after $R$. rickettsii inoculation and lasted until the end of the experiment (Fig. 4B). Maximum temperatures in CGPs were between $40.5^{\circ} \mathrm{C}$ and $41.2^{\circ} \mathrm{C}$. On the last day, mean temperature of IGPs and CGPs was $38.5^{\circ} \mathrm{C}\left(95 \% \mathrm{CI}\right.$ : $\left.38.0-39.0^{\circ} \mathrm{C}\right)$ and $40.4{ }^{\circ} \mathrm{C}\left(95 \% \mathrm{CI}: 39.9-41.0^{\circ} \mathrm{C}\right)$, respectively, and this difference was statistically significant $(T=8.2 ; p=0.0002)$.

All CGPs but no IGPs exhibited behavioral alterations including loss of appetite, increased aggression, weakness, and decreased response to auditory and mechanical stimuli. According to ethical considerations and following the veterinarian's recommendation, the experiment ended on day 11 after infection with $R$. rickettsii because it was advised to euthanize two of the CGPs.

At the end of the experiment, none of the IGPs showed behavioral or macroscopic alterations of the organs that could be attributed to a rickettsial infection, with the exception of one guinea pig that had slightly enlarged testicles. In contrast, the CGPs had severe cachexia, orchitis with dilated blood vessels, abnormal testicular color, and vasodilation in lungs and spleen, as well as several other organs. The presence of $R$. rickettsii was demonstrated by PCR only in testicles of all CGPs. Other organs of CGPs were PCRnegative, as were all organs from IGPs.

In IGPs, the end point titer of IgG antibodies at the end of the experiment was 1:2048 for $R$. rickettsii and 1:1024 for 'Candidatus R. amblyommii'. In the control group, IgG end point titers to $R$. rickettsii were 1:4096 and only 1:512 to R. amblyommii (Fig. 3). As with the previous experiment, all guinea pigs within the same group showed the same end point titers on each day evaluated. 


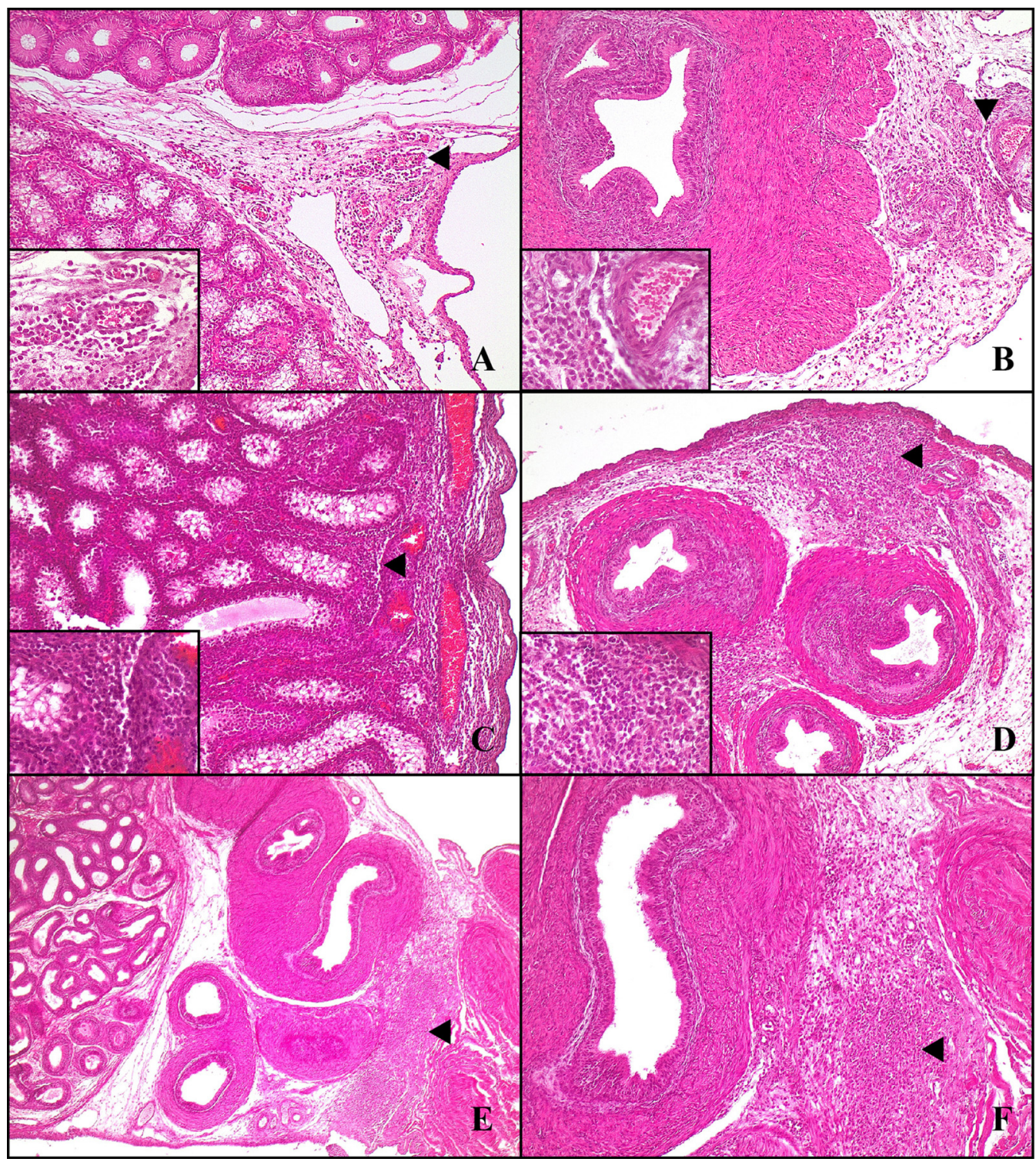

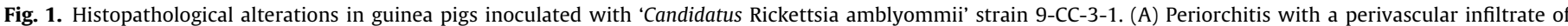

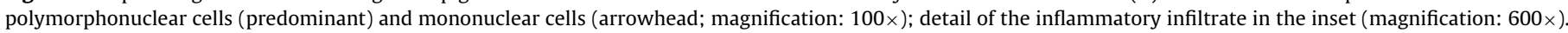

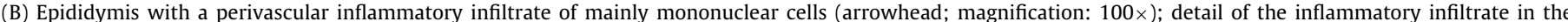

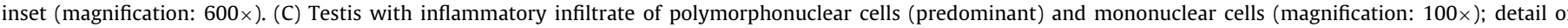

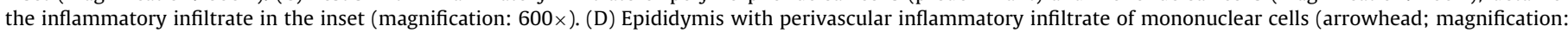

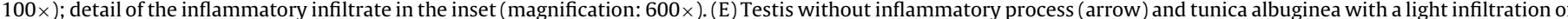

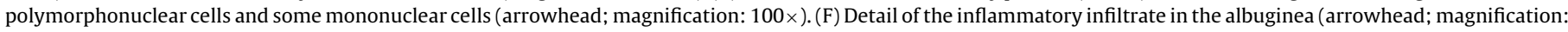
$400 \times)$.

\section{Discussion and conclusions}

In this study, two of the five guinea pigs inoculated with 'Candidatus R. amblyommii' strain 9-CC-3-1 showed signs of testicular alterations early after infection, which was confirmed during necropsies and in the histopathological analysis. In addition, the detection of 'Candidatus R. amblyommii' DNA confirmed that rickettsiae were present in the testicles on day 2, but probably not in other organs. This tropism is common in $R$. rickettsii and other rickettsiae of the spotted fever group (Walker et al., 1977), but had not been demonstrated for 'Candidatus R. amblyommii'. Moreover, it is likely that 'Candidatus R. amblyommii' was responsible for the periorchitis and orchitis, especially considering the presence of a perivascular inflammatory infiltrate in the epididymis inflammatory process. In contrast, no clinical manifestations were observed in guinea pigs independent of the inoculation method in the first studies by Burgdorfer et al. (1981), although rickettsial infection was noted occasionally in tunica vaginalis of meadow voles (M. pennsylvanicus). Similarly in a more recent experiment with guinea pigs, there was no evidence of illness after intradermal and intraperitoneal inoculation with 'Candidatus R. amblyommii' (North Texas isolate), but a histopathological analysis was not performed (Blanton et al., 2014). The pathology observed in the present study may be due to a four times greater amount of bacteria inoculated $\left(4 \times 10^{6}\right)$, when compared to the inoculum used by Blanton et al. (2014) $\left(1 \times 10^{6}\right)$, although both were in the same order of magnitude. While intraperitoneal inoculation was also evaluated by Blanton et al. (2014), it is possible that this infection route may facilitate the entry of bacteria into the testicular tunica vaginalis (Quezada Domínguez, 1997; Pham et al., 2005). 


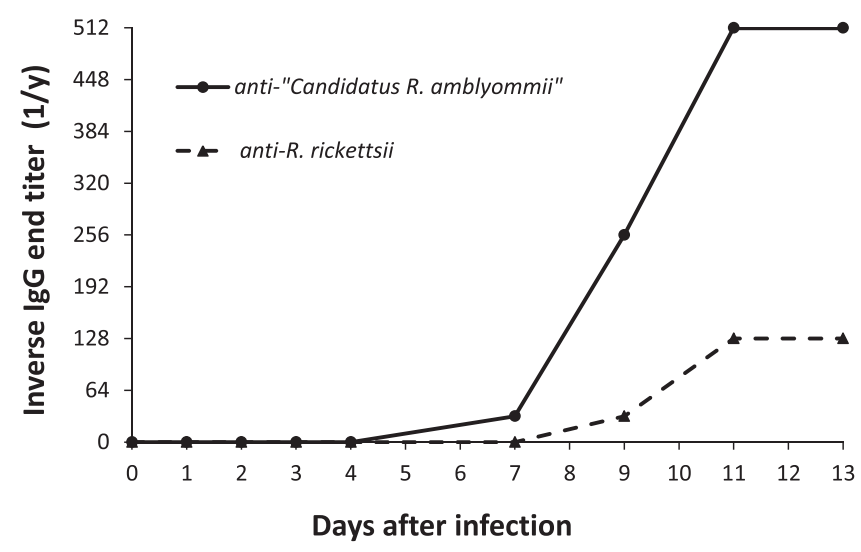

Fig. 2. Anti-Rickettsia IgG inverse end point titers after inoculation of guinea pigs with 'Candidatus Rickettsia amblyommii' strain 9-CC-3-1.

In spite of developing orchitis, guinea pigs inoculated with 'Candidatus R. amblyommii' strain 9-CC-3-1 did not present other signs of disease such as high fever, weight loss, or behavioral abnormalities. Infection with spotted fever group rickettsiae can range from asymptomatic to severe disease, depending on the efficiency

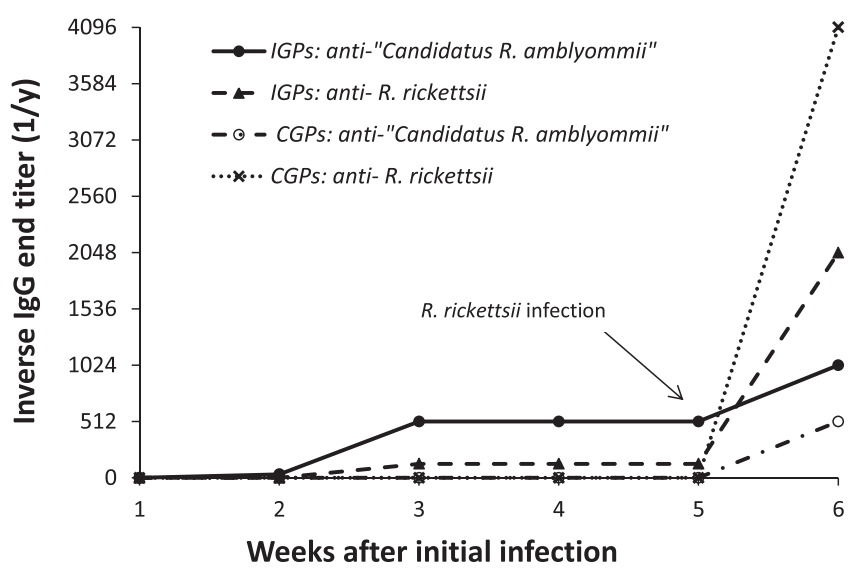

Fig. 3. Anti-Rickettsia IgG inverse end point titers of guinea pigs immunized with 'Candidatus Rickettsia amblyommii' strain 9-CC-3-1 (IGPs) and controls (CGPs) during the assay of cross-protective immunity against Rickettsia rickettsii.

of host's immune response and/or the ability of bacteria to generating disease, which may depend on the strain (Walker, 2007). In concordance with Blanton et al. (2014), guinea pigs in this study developed an immune response evidenced by production

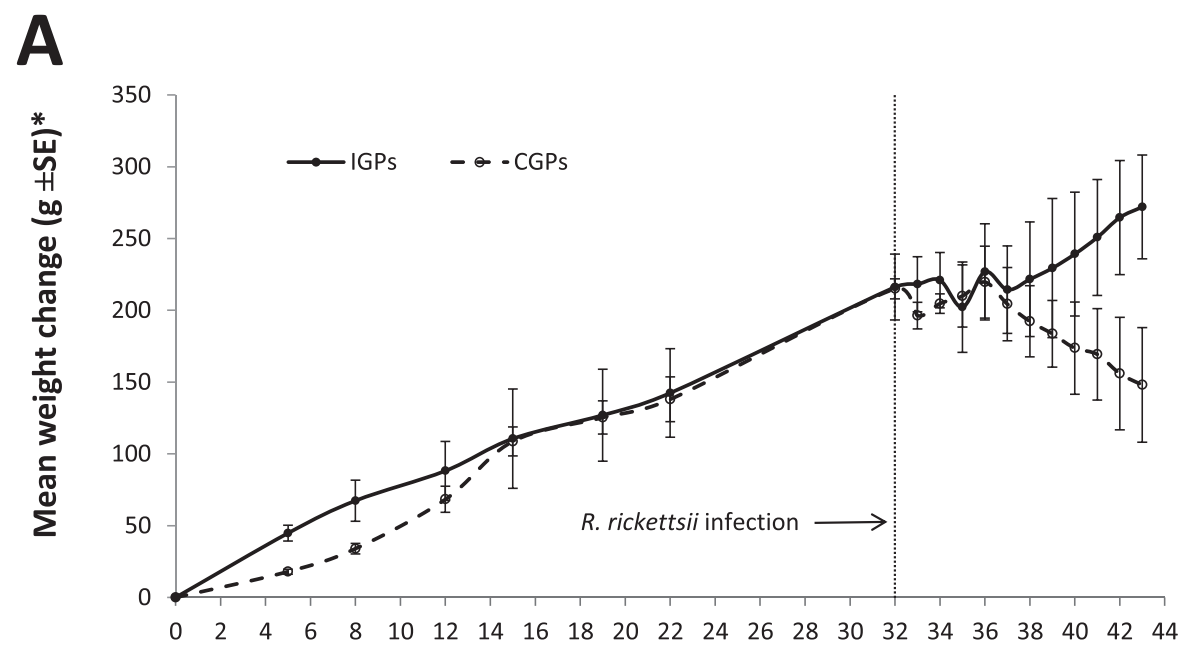

B

\section{Days after initial infection}

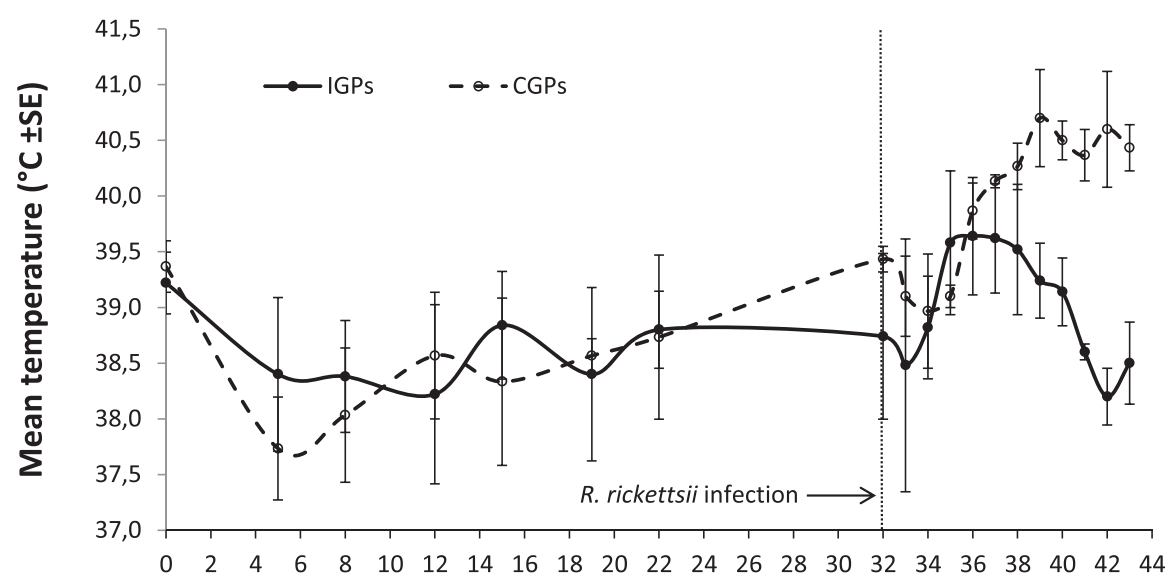

Days after initial infection

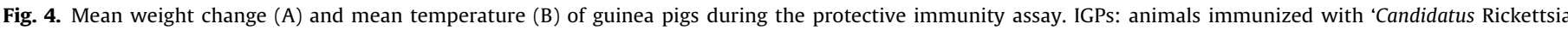
amblyommii' strain 9-CC-3-1; CGPs: Control animals not immunized with 'Candidatus R. amblyommii'; SE: standard error.

Please cite this article in press as: Rivas, J.J., et al., Pathogenic potential of a Costa Rican strain of 'Candidatus Rickettsia amblyommii' in guinea pigs (Cavia porcellus) and protective immunity against Rickettsia rickettsii. Ticks Tick-borne Dis. (2015), http://dx.doi.org/10.1016/j.ttbdis.2015.07.008 
of IgG antibodies, which indicates a possible infection. Although IgG antibodies were detected as early as day 7 after inoculation with 'Candidatus R. amblyommii' strain 9-CC-3-1, maximum IgG end point titers (1:512) were lower than those reported with the North Texas isolate ( $\geq 1: 4096$ at day 14 ) (Blanton et al., 2014). This may be associated with differences in the secondary antibody conjugate used, inoculum size, inoculation route, Rickettsia strain, and characteristics of the guinea pigs. Seroprevalence studies in dogs and horses have shown that IgG end point titers specifically to 'Candidatus $\mathrm{R}$. amblyommii' are variable, but can be as high as 1:4096 (Labruna et al., 2007; Bermúdez et al., 2011). In a prospective study carried out in Oklahoma, USA, dogs that were naturally exposed to ticks seroconverted to 'Candidatus R. amblyommii' without signs of disease and reached maximum IgG end point titers of 1:4096 or greater (Barrett et al., 2014). Therefore, all the recent available evidence suggests that 'Candidatus R. amblyommii' has the capacity to infect and elicit an immune response in guinea pigs, and possibly other animals, although this results in an unapparent or only mild disease.

The assays to determine cross-protective immunity confirmed that a previous infection with 'Candidatus R. amblyommii' strain 9-CC-3-1 generates an immune response that protects against severe disease by R. rickettsii. This was evaluated only recently with 'Candidatus R. amblyommii' North Texas isolate, but it has been studied further in other species to understand protective immune responses and possible candidates for vaccine development (Feng and Waner, 1980; Walker et al., 1984; Feng and Walker, 2003; Valbuena et al., 2004; Blanton et al., 2014). All guinea pigs that had been infected previously with 'Candidatus R. amblyommii' survived developing transient disease after inoculation of $R$. rickettsii, while animals infected only with $R$. rickettsii showed all clinical signs and organ damage that is characteristic of severe disease (Walker et al., 1977; Walker and Henderson, 1978; Arguello et al., 2012). The negative PCR result in most organs of control animals despite their moribund condition suggests a fulminant infection, in which bacteria may have concentrated in the testicles and vascular endothelium but may not have had time to directly infect other organs. Previous studies reported bacteria in spleen, lymph nodes, and testicles (especially in vascular endothelium and smooth muscle) of moribund guinea pigs, but not in other organs (Walker et al., 1977). In addition, there were more advanced lesions and more bacteria when supportive therapy was given as opposed to untreated infections. Considering that endothelial cells are recognized as key immunoreactive cells involved in host defense and inflammation (Sahni et al., 2013), some of the alterations observed in the various organs may be the result of a strong immune response.

The protection observed in immunized guinea pigs demonstrates that there was indeed infection with 'Candidatus $\mathrm{R}$. amblyommii', since previous studies have shown that the immune response obtained from inoculating dead rickettsiae does not prevent animals from dying, independent of antibody production (Kenyon et al., 1979). Moreover, stimulation through the intracellular route that activates $\mathrm{T}$ cells and induces cytokines like IFN- $\gamma$, TNF- $\alpha$, IL-1B, and CCL5 seem to be important in eliminating pathogenic rickettsiae (Feng and Walker, 2000; Valbuena et al., 2004; Sahni et al., 2013). Infection with R. rickettsii probably occurred in guinea pigs previously infected with 'Candidatus R. amblyommii', given transient temperature increase, antibody production, and visible orchitis (one animal), but the resulting immune response efficiently eliminated $R$. rickettsii to levels undetectable by PCR.

In spite of the limitations of this study such as the small number of guinea pigs used and the inoculation dose and route, results show that 'Candidatus R. amblyommii' strain 9-CC-3-1 was able to cause some degree of pathology in guinea pigs, indicating that it should not be excluded as a possible cause of mild disease in these and other animals without further investigation. Indirect evidence has suggested that 'Candidatus R. amblyommii' may be responsible for disease in humans (Billeter et al., 2007; Apperson et al., 2008), thus specific conditions of the host, such as compromised immune system and the presence of other diseases, should be evaluated. Results also demonstrate the immune response generated after infection with 'Candidatus R. amblyommii' strain 9-CC-3-1 from Costa Rica protects guinea pigs from severe disease by a virulent strain of $R$. rickettsii from the same country, which indicates that 'Candidatus $\mathrm{R}$. amblyommii' may modulate local transmission dynamics and the epidemiology of Rocky Mountain Spotted fever in regions where both species are present.

\section{Acknowledgements}

Authors thank Francisco Vega and Carlos Vargas for technical assistance in immunofluorescence assays, rickettsial inoculation, and bacterial quantification; Adrián Avendaño for collaboration during evaluation of guinea pigs and necropsies; and Adrián Avendaño, David H. Walker, Fernando García, and José María Gutiérrez for providing helpful comments and feedback. This investigation was supported by Universidad de Costa Rica project \# 803-B1-041.

\section{References}

Apperson, C.S., Engber, B., Nicholson, W.L., Mead, D.G., Engel, J., Yabsley, M.J., Dail, K., Johnson, J., Watson, D.W., 2008. Tick-borne diseases in North Carolina: is "Rickettsia amblyommii" a possible cause of rickettsiosis reported as Rocky Mountain spotted fever? Vector Borne Zoonotic Dis. 8, 597-606.

Arguello, A.P., Hun, L., Rivera, P., Taylor, L., 2012. A fatal urban case of Rocky Mountain spotted fever presenting an eschar in San José,Costa Rica. Am. J. Trop. Med. Hyg. 87, 345-348.

Barrett, A., Little, S.E., Shaw, E., 2014. "Rickettsia amblyommii" and R. montanensis infection in dogs following natural exposure to ticks. Vector Borne Zoonotic Dis. 14, 20-25.

Bermúdez, C.S.E., Zaldívar, A.Y., Spolidorio, M.G., Moraes-Filho, J., Miranda, R.J., Caballero, C.M., Mendoza, Y., Labruna, M.B., 2011. Rickettsial infection in domestic mammals and their ectoparasites in El Valle de Antón, Coclé, Panamá. Vet. Parasitol. 177, 134-138.

Bermúdez, S.E., Eremeeva, M.E., Karpathy, S.E., Samudio, F., Zambrano, M.L., Zaldivar, Y., Motta, J.A., Dasch, G.A., 2009. Detection and identification of rickettsial agents in ticks from domestic mammals in eastern Panama. J. Med. Entomol. 46, 856-861.

Billeter, S.A., Blanton, H.L., Little, S.E., Levy, M.G., Breitschwerdt, E.B., 2007. Detection of Rickettsia amblyommii in association with a tick bite rash. Vector Borne Zoonotic Dis. 7, 607-610.

Blanton, L.S., Mendell, N.L., Walker, D.H., Bouyer, D.H., 2014. "Rickettsia amblyommii" induces cross protection against lethal Rocky Mountain spotted fever in a guinea pig model. Vector Borne Zoonotic Dis. 14, 557-562.

Burgdorfer, W., Hayes, S., Thomas, L., 1981. A new spotted fever group Rickettsia from the lone star tick. Amblyomma americanum. In: Burgdorfer, W., Anacker, R.L. (Eds.), Rickettsiae and Rickettsial Diseases. Academic Press, New York, pp. 595-602.

CIOMS, ICLAS, 2012. International guiding principles for biomedical research involving animals. Available at: http://www.cioms.ch/images/stories/CIOMS/ IGP2012.pdf (accessed 19.02.15).

Ellison, D.W., Clark, T.R., Sturdevant, D.E., Virtaneva, K., Porcella, S.F., Hackstadt, T., 2008. Genomic comparison of virulent Rickettsia rickettsii Sheila Smith and avirulent Rickettsia rickettsii Iowa. Infect. Immun. 76, 542-550.

Feng, H., Walker, D.H., 2003. Cross-protection between distantly related spotted fever group rickettsiae. Vaccine 21, 3901-3905.

Feng, H.-M., Walker, D.H., 2000. Mechanisms of intracellular killing of Rickettsia conorii in infected human endothelial cells, hepatocytes, and macrophages. Infect. Immun. 68, 6729-6736.

Feng, W.C., Waner, J.L., 1980. Serological cross-reaction and cross-protection in guinea pigs infected with Rickettsia rickettsii and Rickettsia montana. Infect. Immun. 28, 627-629.

Giménez, D.F., 1964. Staining rickettsiae in yolk-sac cultures. Stain Technol. 39 $135-140$.

Hun, L., Troyo, A., Taylor, L., Barbieri, A.M., Labruna, M.B., 2011. First report of the isolation and molecular characterization of Rickettsia amblyommii and Rickettsia felis in Central America. Vector Borne Zoonotic Dis. 11, 1395-1397.

Jiang, J., Yarina, T., Miller, M.K., Stromdahl, E.Y., Richards, A.L., 2010. Molecular detection of Rickettsia amblyommii in Amblyomma americanum parasitizing humans. Vector Borne Zoonotic Dis. 10, 329-340. 
Kenyon, R.H., Kishimoto, R.A., Hall, W.C., 1979. Exposure of guinea pigs to Rickettsia rickettsii by aerosol, nasal, conjunctival, gastric, and subcutaneous routes and protection afforded by an experimental vaccine. Infect. Immun. 25, 580-582.

Labruna, M.B., Horta, M.C., Aguiar, D.M., Cavalcante, G.T., Pinter, A., Gennari, S.M., Camargo, L.M.A., 2007. Prevalence of Rickettsia infection in dogs from the urban and rural areas of Monte Negro Municipality, Western Amazon, Brazil. Vector Borne Zoonotic Dis. 7, 249-255.

Labruna, M.B., Mattar, V., Nava, S., Bermúdez, S., Venzal, S., Dolz, J.M., Abarca, G. Romero, K., de Sousa, L., Oteo, R., Zavala-Castro, J.J., 2011. Rickettsioses in Latin America, Caribbean, Spain and Portugal. Rev. MVZ Córdoba 16, 2435-2457.

Labruna, M.B., Whitworth, T., Horta, M.C., Bouyer, D.H., MacBride, J.W., Pinter, A Popov, V., Gennari, S.M., Walker, D.H., 2004. Rickettsia species infecting Amblyomma cooperi ticks from an area in the State of São Paulo, Brazil, where Brazilian spotted fever is endemic. J. Clin. Microbiol, 42, 90-98.

Luce-Fedrow, A., Wright, C., Gaff, H.D., Sonenshine, D.E., Hynes, W.L., Richards, A.L., 2012. In vitro propagation of Candidatus Rickettsia andeanae isolated from Amblyomma maculatum. FEMS Imunol. Med. Microbiol. 64, 74-81.

Mixson, T.R., Campbell, S.R., Gill, J.S., Ginsberg, H.S., Reichard, M.V., Schulze, T.L. Dasch, G.A., 2006. Prevalence of Ehrlichia, Borrelia, and Rickettsial agents in Amblyomma americanum (Acari: Ixodidae) collected from nine states. J. Med. Entomol. 43, 1261-1268.

Moncayo, A.C., Cohen, S.B., Fritzen, C.M., Huang, E., Yabsley, M.J., Freye, J.D., Dunlap, B.G., Huang, J., Mead, D.G., Jones, T.F., Dunn, J.R., 2010. Absence of Rickettsia rickettsii and occurrence of other spotted fever group rickettsiae in ticks from Tennessee. Am. J. Trop. Med. Hyg. 83, 653-657.

Nadolny, R.M., Wright, C.L., Sonenshine, D.E., Hynes, W.L., Gaff, H.D., 2014. Ticks and spotted fever group rickettsiae of southeastern Virginia. Ticks Tick Borne Dis. 5, 53-57.

Parola, P., Paddock, C.D., Socolovschi, C., Labruna, M.B., Mediannikov, O., Kernif, T. Abdad, M.Y., Stenos, J., Bitam, I., Fournier, P.-E., Raoult, D., 2013. Update on tick-borne rickettsioses around the world: a geographic approach. Clin. Microbiol. Rev, 26, 657-702.

Pham, S.B.T., Hong, M.K.-H., Teague, J.A., Hutson, J.M., 2005. Is the testis intraperitoneal? Pediatr. Surg. Int. 21, 231-239.
Quezada Domínguez, A., 1997. Introducción al manejo de animales de laboratorio: roeadores y pequeñas especies. In: Editorial de la Universidad Autónoma de Yucatán. Merida, Mexico.

Regnery, R.L., Spruill, C.L., Plikaytis, B.D., 1991. Genotypic identification of rickettsiae and estimation of intraspecies sequence divergence for portions of two rickettsial genes. J. Bacteriol. 173, 1576-1589.

Roux, V., Fournier, P.E., Raoult, D., 1996. Differentiation of spotted fever group rickettsiae by sequencing and analysis of restriction fragment length polymorphism of PCR-amplified DNA of the gene encoding the protein rOmpA. J. Clin. Microbiol. 34, 2058-2065.

Sahni, S.K., Narra, H.P., Sahni, A., Walker, D.H., 2013. Recent molecular insights into rickettsial pathogenesis and immunity. Future Microbiol. 8, 1265-1288.

Silverman, D.J., Fiset, P., Wisseman, J.R.C.L., 1979. Simple, differential staining technique for enumerating rickettsiae in yolk sac, tissue culture extracts, or purified suspensions. J. Clin. Microbiol. 9, 437-440.

Valbuena, G., Jordan, J.M., Walker, D.H., 2004. T cells mediate cross-protective immunity between spotted fever group rickettsiae and typhus group rickettsiae. J. Infect. Dis. 190, 1221-1227.

Walker, D.H., 2007. Rickettsiae and rickettsial infections: the current state of knowledge. Clin. Infect. Dis. 45 (Suppl 1), S39-S44.

Walker, D.H., Harrison, A., Henderson, F., Murphy, F.A., 1977. Identification of Rickettsia rickettsii in a guinea pig model by immunofluorescent and electron microscopic techniques. Am. J. Pathol. 86, 343-358.

Walker, D.H., Henderson, F.W., 1978. Effect of immunosuppression on Rickettsia rickettsii infection in guinea pigs. Infect. Immun. 20, 221-227.

Walker, D.H., Montenegro, M.R., Hegarty, B.C., Tringali, G.R., 1984. Rocky Mountain spotted fever vaccine: a regional need. South. Med. J. 77, 447-449.

Wike, D.A., Tallent, G., Peacock, M.G., Ormsbee, R.A., 1972. Studies of the rickettsial plaque assay technique. Infect. Immun. 5, 715-722.

Zhang, X., Ren, X., Norris, D.E., Rasgon, J.L., 2012. Distribution and infection frequency of 'Candidatus Rickettsia amblyommii' in Maryland populations of the lone star tick (Amblyomma americanum) and culture in an Anopheles gambiae mosquito cell line. Ticks Tick Borne Dis. 3, 38-42. 